\title{
Quotient groups of non-nuclear spaces for which the Bochner theorem fails completely
}

by

\author{
Robert STEGLiński (Łódź)
}

\begin{abstract}
It is proved that every real metrizable locally convex space which is not nuclear contains a closed additive subgroup $K$ such that the quotient group $G=(\operatorname{span} K) / K$ admits a non-trivial continuous positive definite function, but no non-trivial continuous character. Consequently, $G$ cannot satisfy any form of the Bochner theorem.
\end{abstract}

Let $G$ be a (Hausdorff) abelian topological group. By a character of $G$ we mean a homomorphism of $G$ into the multiplicative group of complex numbers with modulus 1 . The family of all continuous characters of $G$, with pointwise multiplication and the compact-open topology, is an abelian topological group again. We call it the dual group and denote by $G^{\wedge}$.

A complex-valued function $\varphi$ on $G$ is said to be positive definite if, for all $n \in \mathbb{N}$,

$$
\sum_{i, j=1}^{n} \lambda_{i} \bar{\lambda}_{j} \varphi\left(g_{i}-g_{j}\right) \geq 0
$$

for all $\lambda_{1}, \ldots, \lambda_{n} \in \mathbb{C}$ and $g_{1}, \ldots, g_{n} \in G$. A finite Borel measure $\mu$ on a topological space $X$ is called a Radon measure if, for each Borel subset $A$ and each $\varepsilon>0$, there exists a compact subset $Q$ of $A$ with $\mu(A \backslash Q)<\varepsilon$. Let $\mu$ be a Radon probability measure on $G^{\wedge}$. The characteristic functional of $\mu$, given by

$$
\widehat{\mu}(g)=\int_{G^{\wedge}} \chi(g) d \mu(\chi), \quad g \in G,
$$

is a positive definite function on $G$ with $\widehat{\mu}(0)=1$. We say that $G$ is a $B$-group if it satisfies the Bochner theorem in the following form: for each continuous positive definite function $\varphi$ on $G$ with $\varphi(0)=1$ there is a Radon probability measure $\mu$ on $G^{\wedge}$ with $\widehat{\mu}=\varphi$. The measure $\mu$ is uniquely determined provided that continuous characters separate the points of $G$ (see Theorem 2 in [13]).

2000 Mathematics Subject Classification: 43A35, 43A40, 46A04.

Key words and phrases: Bochner theorem, nuclear spaces, positive definite functions, group characters. 
The Bochner theorem says that every locally compact abelian group is a $B$-group. The Minlos theorem says that every nuclear locally convex space (treated as an additive abelian group) is a $B$-group. The same is true for the so-called nuclear groups, a variety of abelian topological groups containing locally compact abelian groups and nuclear locally convex spaces, introduced in [3] (see Theorem 12.1 in [3] or Theorem 22.16 in [1]; the result obtained there is in fact slightly stronger). On the other hand, if a metrizable locally convex space $G$ is a $B$-group, then $G$ must be a nuclear space (see Theorem 5 , p. 75 in [8]).

There is, however, a certain version of the Bochner theorem which remains valid in all locally convex spaces. Namely, every continuous positive definite function $\varphi$ on a locally convex space $G$ with $\varphi(0)=1$ is the characteristic functional of a (unique) cylindrical probability measure on $G^{\wedge}$, so that it can be in some way synthesized of continuous characters (see Theorem 1, p. 348 in [5] or Proposition A, p. 21 in [9]). The situation becomes completely different if we consider quotient groups.

Let $K$ be a closed subgroup of a topological vector space $E$. Continuous characters separate the points of $E / K$ if and only if $K$ is weakly closed in $E$, and $(E / K)^{\wedge}=\{1\}$ if and only if $K$ is weakly dense in $E$ ([3, Proposition 2.5]). Every closed subgroup of a nuclear locally convex space is weakly closed ([2, Theorem A]). On the other hand, if a real metrizable locally convex space is not nuclear, then it contains a discrete subgroup $K$ such that the quotient group $(\operatorname{span} K) / K$ has no non-trivial continuous positive definite functions, i.e. no non-trivial continuous unitary representations (see [3, Theorem 6.1]).

We say that an abelian topological group $G$ is $N B T$ (no Bochner theorem) if it admits a non-trivial continuous positive definite function $\varphi$, but $G^{\wedge}=\{1\}$. Then $\varphi$ cannot be synthesized of continuous characters (since the latter do not exist), and one cannot speak of any version of the Bochner theorem in this case.

Throughout the paper, we assume that all vector spaces are over the real field $\mathbb{R}$, unless explicitly stated otherwise. Consider the Banach space $L^{p}(0,1), 1 \leq p<\infty$, and let $L_{\mathbb{Z}}^{p}(0,1)$ be the closed subgroup of $L^{p}(0,1)$ consisting of integer-valued functions. Then $L_{\mathbb{Z}}^{p}(0,1)$ is weakly dense in $L^{p}(0,1)$ and the quotient group $L^{p}(0,1) / L_{\mathbb{Z}}^{p}(0,1)$ is NBT (cf. Lemma 5 below). Simple examples of NBT quotient groups of $l^{p}, 1<p \leq 2$, are given by Theorem 5.1(c) and (e) in [3]. It has been proved in [12] that every infinite-dimensional real normed space contains a discrete subgroup $K$ such that the quotient group $(\operatorname{span} K) / K$ is NBT. It was conjectured in [3, p. 111] that NBT quotient groups can be constructed in every non-nuclear locally convex metrizable space. The aim of the present paper is to prove that conjecture. 
THEOREM 1. Every real metrizable locally convex space which is not nuclear contains a closed additive subgroup $K$ such that the quotient group $G=(\operatorname{span} K) / K$ is $N B T$.

The proof will be preceded by several lemmas. By $\lambda_{n}$ we denote the Lebesgue measure on $\mathbb{R}^{n}$. Let $U, W$ be two symmetric convex bodies in an $n$-dimensional vector space $X$. Let $\frac{|U|}{|W|}$ denote the real number defined by

$$
\frac{|U|}{|W|}:=\frac{\lambda_{n}(T(U))}{\lambda_{n}(T(W))}
$$

where $T: X \rightarrow \mathbb{R}^{n}$ is a linear isomorphism.

Lemma 2. Let $U, W$ be two symmetric convex bodies in an $n$-dimensional vector space $N$ with $U \subset W$. Let $M$ be an m-dimensional subspace of $N$ and $\pi: N \rightarrow M$ an arbitrary projection. Then

$$
\begin{gathered}
\frac{|U \cap M|}{|W \cap M|} \geq \frac{m !}{n !} \frac{|U|}{|W|}, \\
\frac{|\pi(U)|}{|\pi(W)|} \geq \frac{m !}{n !} \frac{|U|}{|W|} .
\end{gathered}
$$

For (a), see [3, Lemma 6.6]. The proof of (b) is similar; we leave it to the reader.

Let $p$ be a seminorm on a vector space $E$. The quotient space $E / p^{-1}(0)$ with the canonical norm will be denoted by $E_{p}$. If $q \leq p$ is another seminorm on $E$, then the canonical operator $E_{p} \rightarrow E_{q}$ will be denoted by $T_{p q}$.

Let $T: E \rightarrow F$ be a bounded linear operator between normed spaces. For each $n=1,2, \ldots$, we define

$$
\nu_{n}(T)=\sup _{M}\left(\frac{\left|T\left(B_{E} \cap M\right)\right|}{\left|B_{F} \cap T(M)\right|}\right)^{1 / n}
$$

where $B_{E}, B_{F}$ denote the closed unit balls in $E, F$ respectively and the supremum is taken over all linear subspaces $M$ of $E$ with $\operatorname{dim} M=\operatorname{dim} T(M)=n$. If $\operatorname{rank} T<n$, then we define $\nu(T)=0$.

Lemma 3. Let $E$ be a locally convex space. Suppose that there exists an $\varepsilon>0$ with the following property: for each continuous seminorm $q$ on $E$ there is another continuous seminorm $p \geq q$ such that $\nu_{n}\left(T_{p q}\right)=o\left(n^{-\varepsilon}\right)$. Then $E$ is nuclear.

This is Lemma 6.5 of [3].

Lemma 4. Let $E, F$ be normed spaces and let $T: E \rightarrow F$ be an injective bounded linear operator such that

$$
\limsup _{n \rightarrow \infty} n^{1 / 5} \nu_{n}(T)=\infty
$$


If $X$ is a subspace of $E$ with $\operatorname{codim} X<\infty$, then

$$
\limsup _{n \rightarrow \infty} n^{1 / 5} \nu_{n}\left(T_{\mid X}\right)=\infty .
$$

The proof of this lemma is similar to that of Lemma 6.8 of [3].

Lemma 5. Let $K$ be a closed subgroup of a topological vector space $E$ and let $\sigma: E \rightarrow E / K$ be the canonical homomorphism.

(a) Let $T: E \rightarrow L^{2}(0,1)$ be a non-zero continuous linear operator with $T(K) \subset L_{\mathbb{Z}}^{2}(0,1)$. Then the formula

$$
\varphi(x)=\int_{0}^{1} \exp \{2 \pi i T x(t)\} d t, \quad x \in E,
$$

defines a non-trivial continuous positive definite function $\varphi$ on $E$ with $\varphi \equiv 1$ on $K$. Consequently, the formula $\psi(\sigma(x))=\varphi(x), x \in E$, defines a non-trivial continuous positive definite function $\psi$ on $E / K$.

(b) Let $\chi$ be a non-trivial continuous character of $E / K$. Then there exists a non-zero continuous linear functional $f$ on $E$ with $f(K) \subset \mathbb{Z}$ such that

$$
\chi(\sigma(x))=\exp \{2 \pi i f(x)\} \quad \text { for each } x \in E .
$$

Proof. (a) It is not hard to see that

$$
\Phi_{x} f(t)=f(t) \cdot \exp \{2 \pi i T x(t)\} \quad\left(x \in E ; f \in L^{2}(0,1) ; t \in(0,1)\right)
$$

defines a continuous unitary representation $\Phi$ of the group $E$ in the complex Hilbert space $L^{2}(0,1)$ (see e.g. the proof of Proposition 4.1 in [3]). Let $f_{0} \equiv 1$ on $(0,1)$. Then we have $\varphi(x)=\left(\Phi_{x} f_{0}, f_{0}\right)$ for each $x \in E$, which means that $\varphi$ is a continuous positive definite function on $E$. Since $T \not \equiv 0$, there is some $x \in E$ with $T x \notin L_{\mathbb{Z}}^{2}(0,1)$, and then $\operatorname{Re} \varphi(x)<1$. The last assertion is standard (see e.g. [6, (32.6)]).

(b) The composition $\chi \circ \sigma$ is a continuous character of $E$. Therefore there exists a continuous linear functional $f$ on $E$ satisfying $(*)$ (see e.g. $[11$, Lemma 1], or $[6,(23.32)]$, or $[3,(2.3)])$. It is clear that $f(K) \subset \mathbb{Z}$.

By a step function we mean a linear combination of characteristic functions of finite intervals. By $S_{\mathbb{Z}}(0,1)$ we denote the set of integer-valued step functions on $(0,1)$.

Lemma 6. Let $I$ be a finite interval and let $\alpha \in \mathbb{R}$. Then there exists a step function $\psi: I \rightarrow(-1,1)$ such that $\psi+\alpha$ is integer-valued and $\int_{I} \psi=0$.

Proof. Let $I=(a, b)$. If $\alpha \in \mathbb{Z}$, we set $\psi \equiv 0$. Suppose $\alpha \notin \mathbb{Z}$. Then

$$
c:=a+(b-a)(\alpha-[\alpha]) \in(a, b)
$$


and we may define, for instance,

$$
\psi(t)= \begin{cases}{[\alpha]-\alpha+1} & \text { for } t \in(a, c), \\ {[\alpha]-\alpha} & \text { for } t \in(c, b) .\end{cases}
$$

Let $X, Y$ be two $n$-dimensional normed spaces. Their Banach-Mazur distance will be denoted by $d(X, Y)$. By $\mathbb{R}_{2}^{n}$ we denote the space $\mathbb{R}^{n}$ endowed with the canonical euclidean norm. We will write $B_{2}^{n}$ for the closed unit ball of $\mathbb{R}_{2}^{n}$.

We will need the Milman quotient subspace theorem in the following form (see Theorem 3.1.1, p. 1171 in [7]):

LEMMA 7. Let $1 / 2 \leq \alpha \leq 1$ and let $X$ be a normed space of dimension $n$. Then there exist subspaces $E \supset F$ of $X$ with

$$
k=\operatorname{dim} E / F \geq \alpha n, \quad d\left(E / F, \mathbb{R}_{2}^{k}\right)<r
$$

for some constant $r$ independent of $n$.

Let $E$ be a vector space and let $A \subset E$. The linear subspace and the additive subgroup generated by $A$ are denoted by $\langle A\rangle$ and $\langle A\rangle_{\mathbb{Z}}$, respectively.

Let $E$ be a normed space. The adjoint space of $E$ will be denoted by $E^{*}$. We say that a closed subgroup $K$ of $E$ is finite-dimensional if $\operatorname{dim}\langle K\rangle<\infty$. Then the group $K$ is topologically isomorphic to $\mathbb{R}^{a} \times \mathbb{Z}^{b}$, where $a$ and $b$ are non-negative integers (see Theorem (9.11) in [6]).

Let $\|\cdot\|_{0},\|\cdot\|_{1}$ be two norms on a vector space $E$. Write

$$
E_{0}:=\left(E,\|\cdot\|_{0}\right), \quad E_{1}:=\left(E,\|\cdot\|_{1}\right)
$$

and

$$
B_{0}:=B_{E_{0}}, \quad B_{1}:=B_{E_{1}} .
$$

Let $M, N$ be two finite-dimensional subspaces of $E$. Let $M_{0}\left(\operatorname{resp} .(M / N)_{0}\right)$ denote the space $M(\operatorname{resp} . M / N)$ endowed with the norm induced by $\|\cdot\|_{0}$. Let $M_{1}$ (resp. $\left.(M / N)_{1}\right)$ denote the space $M($ resp. $M / N)$ endowed with the norm induced by $\|\cdot\|_{1}$. If $T: M \rightarrow L^{2}(0,1)$ is a linear operator, then $\|T\|_{0}$ denotes the norm of $T: M_{0} \rightarrow L^{2}(0,1)$. If $f: M \rightarrow \mathbb{R}(\operatorname{resp} . f: M / N \rightarrow \mathbb{R})$ is a linear functional, then $\|f\|_{1}$ denotes the norm of $f: M_{1} \rightarrow \mathbb{R}$ (resp. $\left.f:(M / N)_{1} \rightarrow \mathbb{R}\right)$.

LEMMA 8. Let $\|\cdot\|_{0},\|\cdot\|_{1}$ be two norms on a vector space $E$ such that $\|\cdot\|_{0} \leq\|\cdot\|_{1}$ and let $I: E_{1} \rightarrow E_{0}$ denote the identity operator. Suppose that

$$
\limsup _{n \rightarrow \infty} n^{1 / 5} \nu_{n}(I)=\infty \text {. }
$$

Let $K_{1}, K_{2}$ be finite-dimensional closed subgroups of $E$ with $\left\langle K_{1}\right\rangle \cap\left\langle K_{2}\right\rangle$ $=\{0\}$ and let $K=K_{1}+K_{2}$. Let $f:\left\langle K_{1}\right\rangle \rightarrow \mathbb{R}$ be a non-zero linear functional with $f\left(K_{1}\right) \subset \mathbb{Z}$ and let $T:\langle K\rangle \rightarrow L^{2}(0,1)$ be a non-zero linear operator with $T(K) \subset S_{\mathbb{Z}}(0,1)$. 
Given $\varepsilon \in(0,1)$, one can find a finite-dimensional closed subgroup $K_{3}$ such that $\langle K\rangle \cap\left\langle K_{3}\right\rangle=\{0\}$ and if we define $\widetilde{K}=K+K_{3}$, then the following conditions are satisfied:

(i) there are no linear functionals $\tilde{f}:\langle\widetilde{K}\rangle \rightarrow \mathbb{R}$ with $\widetilde{f}_{\mid\left\langle K_{1}\right\rangle}=f$ and $\widetilde{f}(\widetilde{K}) \subset \mathbb{Z}$ such that $\|\widetilde{f}\|_{1} \leq 1$;

(ii) there exists a linear operator $\widetilde{T}:\langle\widetilde{K}\rangle \rightarrow L^{2}(0,1)$ with $\widetilde{T}_{\langle K\rangle}=T$ and $\widetilde{T}(\widetilde{K}) \subset S_{\mathbb{Z}}(0,1)$ such that $\|\widetilde{T}\|_{0} \leq(1+\varepsilon)\|T\|_{0}$.

Proof. Fix $\varepsilon \in(0,1)$. Choose $\delta \in(0,1)$ such that

$$
\frac{1}{1-\delta}<1+\frac{\varepsilon}{2}
$$

A standard argument shows that there is a linear subspace $X \subset E$ with $\operatorname{codim} X<\infty$ such that

$$
\|x+y\|_{0} \geq(1-\delta)\|x\|_{0} \quad \text { for all } x \in\langle K\rangle \text { and } y \in X .
$$

Then $\langle K\rangle \cap X=\{0\}$. Moreover, we have

$$
\|x+y\|_{0} \geq \frac{1-\delta}{2-\delta} \cdot\|y\|_{0} \quad \text { for all } x \in\langle K\rangle \text { and } y \in X .
$$

Let $A=\sqrt{2 \pi} e(5 / 4)^{2}$. Let $r$ be the constant corresponding to $\alpha=4 / 5$ in Lemma 7. Choose $\gamma>0$ such that

$$
\begin{gathered}
\frac{2-\delta}{1-\delta} \cdot \frac{r}{\gamma} \leq \frac{\varepsilon}{2}\|T\|_{0}, \\
A \cdot \gamma \geq 1 .
\end{gathered}
$$

From (1) and Lemma 4 it follows that

$$
\limsup _{n \rightarrow \infty} n^{1 / 5} \nu_{n}\left(I_{\mid X}\right)=\infty .
$$

Therefore we can find some $n$ and an $n$-dimensional subspace $Y$ of $X$ such that

$$
\frac{\left|B_{1} \cap Y\right|}{\left|B_{0} \cap Y\right|}>(A \gamma)^{n} n^{-n / 5}
$$

According to our definition of $r$, we can find a subspace $M$ of $Y$ and a subspace $N$ of $M$ such that

$$
\begin{gathered}
l:=\operatorname{dim}(M / N)>\frac{4}{5} n \\
d\left((M / N)_{0}, \mathbb{R}_{2}^{l}\right)<r
\end{gathered}
$$


Let $\pi: M \rightarrow M / N$ be the canonical projection. Set $m=\operatorname{dim} M$. Applying Lemma 2 (a) and then (b), we see that

$$
\frac{\left|\pi\left(B_{1} \cap M\right)\right|}{\left|\pi\left(B_{0} \cap M\right)\right|} \geq \frac{l !}{m !} \frac{\left|B_{1} \cap M\right|}{\left|B_{0} \cap M\right|} \geq \frac{l !}{m !} \frac{m !}{n !} \frac{\left|B_{1} \cap Y\right|}{\left|B_{0} \cap Y\right|} .
$$

Hence, by (6)-(8) and Stirling's formula, we derive

$$
\begin{aligned}
\frac{\left|\pi\left(B_{1} \cap M\right)\right|}{\left|\pi\left(B_{0} \cap M\right)\right|} & \geq \frac{l !}{n !} \frac{\left|B_{1} \cap Y\right|}{\left|B_{0} \cap Y\right|}>\frac{l !}{n !}(A \gamma)^{n} n^{-n / 5} \\
& \geq \frac{\sqrt{2 \pi l}\left(\frac{l}{e}\right)^{l}}{\sqrt{2 \pi \frac{5}{4} l}\left(\frac{5 l}{4 e}\right)^{5 l / 4} e} \cdot(A \gamma)^{l} l^{-l / 4}\left(\frac{4}{5}\right)^{l / 4} \\
& \geq \gamma^{l}\left(\frac{2 \pi e}{l}\right)^{l / 2} \geq \gamma^{l} \lambda_{l}\left(B_{2}^{l}\right) .
\end{aligned}
$$

It follows from (9) that there is a linear isomorphism $R: \mathbb{R}_{2}^{l} \rightarrow(M / N)_{0}$ such that $\|R\| \leq 1$ and $\left\|R^{-1}\right\|<r$. Let $R^{*}:(M / N)_{0}^{*} \rightarrow \mathbb{R}_{2}^{l}$ be the adjoint operator (we identify $\left(\mathbb{R}_{2}^{l}\right)^{*}$ with $\mathbb{R}_{2}^{l}$ in the usual way). Put $S=\gamma R^{*}$. Then $\left\|S^{*}\right\|=\gamma\|R\| \leq \gamma$, i.e.

$$
\left(S^{*}\right)^{-1}\left(B_{(M / N)_{0}}\right) \supset \gamma^{-1} B_{2}^{l} .
$$

Since $\left(S^{*}\right)^{-1}$ is a linear isomorphism, we have

$$
\frac{\left|\pi\left(B_{1} \cap M\right)\right|}{\left|\pi\left(B_{0} \cap M\right)\right|}=\frac{\lambda_{l}\left(\left(S^{*}\right)^{-1}\left(B_{(M / N)_{1}}\right)\right)}{\lambda_{l}\left(\left(S^{*}\right)^{-1}\left(B_{(M / N)_{0}}\right)\right)} .
$$

Let $B_{(M / N)_{1}^{*}}$ denote the closed unit ball in the adjoint space of $(M / N)_{1}$. The sets $S\left(B_{\left.(M / N)_{1}^{*}\right)}\right.$ and $\left(S^{*}\right)^{-1}\left(B_{(M / N)_{1}}\right)$ are polar reciprocal to each other with respect to the scalar product in $\mathbb{R}_{2}^{l}$. Therefore, by the Santaló inequality (see (4.3.5) in [7] or $\S 4$ in [10]), we have

$$
\lambda_{l}\left(S\left(B_{(M / N)_{1}^{*}}\right)\right) \cdot \lambda_{l}\left(\left(S^{*}\right)^{-1}\left(B_{(M / N)_{1}}\right)\right) \leq\left(\lambda_{l}\left(B_{2}^{l}\right)\right)^{2} .
$$

Now, from (10)-(13), we get

$$
\lambda_{l}\left(S\left(B_{(M / N)_{1}^{*}}\right)\right)<1 .
$$

Let $e_{1}, \ldots, e_{l}$ be the canonical orthonormal basis in $\mathbb{R}_{2}^{l}$ and let $\mathbb{Z}^{l}=\mathbb{Z} e_{1}+$ $\cdots+\mathbb{Z} e_{l}$ be the integer lattice. From (14) it follows that $S\left(B_{(M / N)_{1}^{*}}\right)+\mathbb{Z}^{l} \neq \mathbb{R}^{l}$. So, there is some $\xi=\left(\xi_{1}, \ldots, \xi_{l}\right) \in \mathbb{R}^{l}$ such that

$$
\left(\mathbb{Z}^{l}-\xi\right) \cap S\left(B_{(M / N)_{1}^{*}}\right)=\emptyset .
$$

The vectors $w_{i}=\gamma R\left(e_{i}\right), i=1, \ldots, l$, form a basis in $M / N$. Choose $b_{i} \in M$ such that $\pi\left(b_{i}\right)=w_{i}$. Choose $v \in\left\langle K_{1}\right\rangle$ with $f(v)=1$. Let

$$
K_{3}=N+\left\langle b_{1}+\xi_{1} v, \ldots, b_{l}+\xi_{l} v\right\rangle_{\mathbb{Z}} .
$$


Then $K_{3}$ is a finite-dimensional closed subgroup of $E$. It is easy to see that $\langle K\rangle \cap\left\langle K_{3}\right\rangle=\{0\}$ and $\langle K\rangle+\left\langle K_{3}\right\rangle=\langle K\rangle+M$. Define

$$
\widetilde{K}=K+K_{3} .
$$

Then $\widetilde{K}$ is a finite-dimensional closed subgroup of $E$ with $K \subset \widetilde{K}$.

To prove (i), take an arbitrary $\widetilde{f} \in\langle\widetilde{K}\rangle^{*}$ with $\widetilde{f}_{\mid\left\langle K_{1}\right\rangle}=f$ and $\widetilde{f}(\widetilde{K}) \subset \mathbb{Z}$. Since $N \subset \widetilde{K}$, it follows that $\widetilde{f}(N)=\{0\}$, and hence there is a linear functional $g \in(M / N)^{*}$ with $\widetilde{f}_{\mid M}=g \circ \pi$ and $\|g\|_{1}=\left\|\widetilde{f}_{\mid M}\right\|_{1}$. It is easy to check that

$$
S(h)=\sum_{i=1}^{l} h\left(w_{i}\right) e_{i} \quad \text { for all } h \in(M / N)^{*} .
$$

Hence

$$
\begin{aligned}
S(g) & =\sum_{i=1}^{l} g\left(w_{i}\right) e_{i}=\sum_{i=1}^{l} g\left(\pi\left(b_{i}\right)\right) e_{i}=\sum_{i=1}^{l} \widetilde{f}_{\mid M}\left(b_{i}\right) e_{i} \\
& =\sum_{i=1}^{l} \widetilde{f}\left(b_{i}+\xi_{i} v\right) e_{i}-\sum_{i=1}^{l} \widetilde{f}\left(\xi_{i} v\right) e_{i} \in \mathbb{Z}^{l}-\xi .
\end{aligned}
$$

In view of $(15)$, this means that $g \notin B_{(M / N)_{1}^{*}}$, or equivalently, $\|g\|_{1}>1$, which gives $\|\widetilde{f}\|_{1} \geq\left\|\widetilde{f}_{\mid M}\right\|_{1}=\|g\|_{1}>1$.

To prove (ii), we shall construct a sequence $\varphi_{1}, \ldots, \varphi_{l}$ of pairwise orthogonal step functions on $(0,1)$ such that $\left|\varphi_{i}\right| \leq 1$ and

$$
\varphi_{i}+\xi_{i} T v \in S_{\mathbb{Z}}(0,1) \quad \text { for } i=1, \ldots, l .
$$

Since $T(K) \subset S_{\mathbb{Z}}(0,1)$ and $v \in\left\langle K_{1}\right\rangle \subset\langle K\rangle$, it follows that $T v$ is a step function. We may write

$$
T v=\sum_{j=1}^{m_{1}} \alpha_{j} \chi_{I_{j}}
$$

where $\alpha_{1}, \ldots, \alpha_{m_{1}} \in \mathbb{R}$ and $I_{1}, \ldots, I_{m_{1}}$ is a decomposition of $(0,1)$ into some smaller intervals. We define

$$
\varphi_{1}(t)=\left[\xi_{1} \alpha_{j}\right]-\xi_{1} \alpha_{j}
$$

for $t \in I_{j}, j=1, \ldots, m_{1}$. Then $\left|\varphi_{1}\right|<1$ and $\varphi_{1}+\xi_{1} T v \in S_{\mathbb{Z}}(0,1)$.

Then we proceed by induction. Suppose we have constructed $\varphi_{1}, \ldots, \varphi_{k-1}$ for a certain $k=2, \ldots, l$. The interval $(0,1)$ decomposes into a finite union of smaller intervals $I_{j}^{(k)}, 1 \leq j \leq m_{k}$, such that each of the functions $T v, \varphi_{1}, \ldots, \varphi_{k-1}$ is constant on every $I_{j}^{(k)}$. By Lemma 6 , for each $j=1, \ldots, m_{k}$ we can find a step function $\psi_{j}$ on $I_{j}^{(k)}$ with $\left|\psi_{j}\right| \leq 1$ such that $\int_{I_{j}^{(k)}} \psi_{j}=0$ 
and $\psi_{j}+\xi_{k} T v$ is integer-valued. We define

$$
\varphi_{k}(t)=\psi_{j}(t) \quad \text { for } t \in I_{j}^{(k)}, j=1, \ldots, m_{k} .
$$

Then $\varphi_{k}$ is a step function on $(0,1)$ with $\left|\varphi_{k}\right| \leq 1$ such that $\varphi_{k}+\xi_{k} T v \in$ $S_{\mathbb{Z}}(0,1)$. It is clear that $\int_{0}^{1} \varphi_{k} \varphi_{i}=0$ for each $i=1, \ldots, k-1$.

Consider the linear operator $Q: \mathbb{R}_{2}^{l} \rightarrow L^{2}(0,1)$ given by $Q\left(e_{i}\right)=\varphi_{i}$ for $i=1, \ldots, l$. Since the functions $\varphi_{i}$ are pairwise orthogonal and $\left|\varphi_{i}\right| \leq 1$, we have $\|Q\| \leq 1$. Let $T_{1}=\gamma^{-1} Q \circ R^{-1} \circ \pi: M \rightarrow L^{2}(0,1)$. Then $T_{1} b_{i}=\varphi_{i}$ for $i=1, \ldots, l$. Since $\left\|R^{-1}\right\|<r$, we have $\left\|T_{1}\right\|_{0}<r \gamma^{-1}$. Let $P_{1}:\langle\widetilde{K}\rangle_{0} \rightarrow M_{0}$ and $P_{2}:\langle\widetilde{K}\rangle_{0} \rightarrow\langle K\rangle_{0}$ be the projections. By (3) and (4), we have

$$
\left\|P_{1}\right\| \leq \frac{2-\delta}{1-\delta}, \quad\left\|P_{2}\right\| \leq \frac{1}{1-\delta} .
$$

Define $\widetilde{T}=T_{1} P_{1}+T P_{2}:\langle\widetilde{K}\rangle \rightarrow L^{2}(0,1)$. Then $\widetilde{T}_{\mid\langle K\rangle}=T$. In view of (2), (5), (18), we have

$$
\begin{aligned}
\|\widetilde{T}\|_{0} & \leq\left\|T_{1}\right\|_{0}\left\|P_{1}\right\|+\|T\|_{0}\left\|P_{2}\right\| \\
& \leq \gamma^{-1} r \cdot \frac{2-\delta}{1-\delta}+\frac{1}{1-\delta} \cdot\|T\|_{0} \leq(1+\varepsilon)\|T\|_{0} .
\end{aligned}
$$

To finish the proof we must show that $\widetilde{T}(\widetilde{K}) \subset S_{\mathbb{Z}}(0,1)$. Since $\widetilde{K}=$ $K+N+\left\langle b_{1}+\xi_{1} v, \ldots, b_{l}+\xi_{l} v\right\rangle_{\mathbb{Z}}$, it suffices to make the following observation: if $x \in K$, then $\widetilde{T}(x)=T(x) \in S_{\mathbb{Z}}(0,1)$; if $x \in N$, then $\widetilde{T}(x)=0$; and if $x=b_{i}+\xi_{i} v$ for some $i=1, \ldots, l$, then

$$
\widetilde{T}\left(b_{i}+\xi_{i} v\right)=T\left(\xi_{i} v\right)+\left(Q \circ R^{-1} \circ \pi\right)\left(b_{i}\right)=\xi_{i} T v+\varphi_{i} \in S_{\mathbb{Z}}(0,1)
$$

by (17).

Lemma 9. Let $\|\cdot\|_{0},\|\cdot\|_{1}$ be two norms on a vector space $E$ such that $\|\cdot\|_{0} \leq\|\cdot\|_{1}$ and let $I: E_{1} \rightarrow E_{0}$ denote the identity operator. Suppose that

$$
\limsup _{n \rightarrow \infty} n^{1 / 5} \nu_{n}(I)=\infty .
$$

Let $K$ be a finite-dimensional closed subgroup of $E$ and let $T:\langle K\rangle \rightarrow$ $L^{2}(0,1)$ be a non-zero linear operator with $T(K) \subset S_{\mathbb{Z}}(0,1)$. Then, given $\varepsilon \in(0,1)$, one can find a finite-dimensional closed subgroup $\widetilde{K}$ with $K \subset \widetilde{K}$ such that the following conditions are satisfied:

(i) there are no linear functionals $\tilde{f}:\langle\widetilde{K}\rangle \rightarrow \mathbb{R}$ with $\widetilde{f}_{\mid\langle K\rangle} \not \equiv 0$ and $\widetilde{f}(\widetilde{K}) \subset \mathbb{Z}$ such that $\|\widetilde{f}\|_{1} \leq 1$;

(ii) there exists a linear operator $\widetilde{T}:\langle\widetilde{K}\rangle \rightarrow L^{2}(0,1)$ with $\widetilde{T}_{\langle K\rangle}=T$ and $\widetilde{T}(\widetilde{K}) \subset S_{\mathbb{Z}}(0,1)$ such that $\|\widetilde{T}\|_{0} \leq(1+\varepsilon)\|T\|_{0}$.

Proof. It is clear that the set

$$
Q=\left\{f \in\langle K\rangle^{*}: f(K) \subset \mathbb{Z} \text { and }\|f\|_{1} \leq 1\right\}
$$


is finite. Let $f_{1}, \ldots, f_{m}$ be the non-zero elements of $Q$ (if there are none, we may just take $\widetilde{K}=K)$. Choose $\varepsilon^{\prime} \in(0,1)$ such that

$$
\left(1+\varepsilon^{\prime}\right)^{m} \leq 1+\varepsilon .
$$

Set $K_{0}=K$. Applying the previous lemma, we find inductively an increasing sequence $K_{0}, K_{1}, \ldots, K_{m}$ of finite-dimensional closed subgroups of $E$ and a sequence of linear operators $T_{i}:\left\langle K_{i}\right\rangle \rightarrow L^{2}(0,1)$ for $i=0,1, \ldots, m$ such that, for each $i=1, \ldots, m$, the following conditions are satisfied:

$\left(\mathrm{a}_{i}\right) \quad T_{i}\left(K_{i}\right) \subset S_{\mathbb{Z}}(0,1)$

(bi $\left.\mathrm{b}_{i}\right) \quad T_{i \mid\left\langle K_{i-1}\right\rangle}=T_{i-1}$;

(ci $\left.c_{i}\right) \quad\left\|T_{i}\right\|_{0} \leq\left(1+\varepsilon^{\prime}\right)\left\|T_{i-1}\right\|_{0}$;

$\left(\mathrm{d}_{i}\right) \quad$ there are no linear functionals $f \in\left\langle K_{i}\right\rangle^{*}$ with $f_{\mid\langle K\rangle}=f_{i}$ and $f\left(K_{i}\right) \subset$ $\mathbb{Z}$ such that $\|f\|_{1} \leq 1$.

Define $\widetilde{K}=K_{m}$ and $\widetilde{T}=T_{m}$.

Condition $\left(\mathrm{a}_{m}\right)$ says that $\widetilde{T}(\widetilde{K}) \subset S_{\mathbb{Z}}(0,1)$. Conditions $\left(\mathrm{b}_{i}\right), i=1, \ldots, m$, imply that $\widetilde{T}_{\mid\langle K\rangle}=T$. Conditions $\left(\mathrm{c}_{i}\right)$ together with (20) yield

$$
\|\widetilde{T}\|_{0}=\left\|T_{m}\right\|_{0} \leq\left(1+\varepsilon^{\prime}\right)^{m}\left\|T_{0}\right\|_{0} \leq(1+\varepsilon)\|T\|_{0} .
$$

To prove (ii), take any $\widetilde{f} \in\langle\widetilde{K}\rangle^{*}$ with $\widetilde{f}_{\mid\langle K\rangle} \not \equiv 0$ and $\widetilde{f}(\widetilde{K}) \subset \mathbb{Z}$. If $\widetilde{f}_{\mid\langle K\rangle} \notin Q$, then $\|\widetilde{f}\|_{1} \geq\left\|\widetilde{f}_{\mid\langle K\rangle}\right\|_{1}>1$. So, suppose that $\widetilde{f}_{\mid\langle K\rangle}=f_{i}$ for a certain $i=1, \ldots, m$. Let $f=\widetilde{f}_{\mid\left\langle K_{i}\right\rangle}$. Then we have $f_{\mid\langle K\rangle}=\widetilde{f}_{\mid\langle K\rangle}=f_{i}$ and $f\left(K_{i}\right)=\widetilde{f}\left(K_{i}\right) \subset \widetilde{f}(\widetilde{K}) \subset \mathbb{Z}$. Condition $\left(\mathrm{d}_{i}\right)$ says that $\|f\|_{1}>1$, whence $\|\widetilde{f}\|_{1} \geq\|f\|_{1}>1$.

Let $\left(\|\cdot\|_{i}\right)_{i=0}^{\infty}$ be a sequence of norms in a vector space $E$. For each $i=0,1,2, \ldots$, let $E_{i}$ denote the normed space $\left(E,\|\cdot\|_{i}\right)$. Let $M$ be a finitedimensional subspace of $E$. If $f: M \rightarrow \mathbb{R}$ is a linear funcional, then $\|f\|_{i}$, $i=0,1,2, \ldots$, denotes the norm of $f:\left(M,\|\cdot\|_{i}\right) \rightarrow \mathbb{R}$.

Lemma 10. Let $E$ be a metrizable locally convex space with topology defined by an increasing sequence of norms $\left(\|\cdot\|_{i}\right)_{i=0}^{\infty}$. For each $i=1,2, \ldots$, let $I_{i}: E_{i} \rightarrow E_{0}$ be the identity operator. Suppose that

$$
\limsup _{n \rightarrow \infty} n^{1 / 5} \nu_{n}\left(I_{i}\right)=\infty
$$

for $i=1,2, \ldots$ Then there exists a closed subgroup $K$ of $E$ such that the quotient group $\langle K\rangle / K$ is $N B T$.

Proof. Set

$$
B_{i}=\left\{x \in E:\|x\|_{i} \leq 1\right\}, \quad i=1,2, \ldots .
$$

Without loss of generality we can assume that $\left\{B_{i}\right\}_{i=1}^{\infty}$ is a neighbourhood base at zero in $E$. 
Choose $\varepsilon_{i} \in(0,1), i=1,2, \ldots$, such that

$$
\prod_{i=1}^{\infty}\left(1+\varepsilon_{i}\right)<\infty .
$$

Next, choose some $u \in E$ with $\|u\|_{0}=1$. Define $K_{0}=\langle u\rangle_{\mathbb{Z}}$ and let $T_{0}:\left\langle K_{0}\right\rangle \rightarrow L^{2}(0,1)$ be the linear operator such that $T_{0} u \equiv 1$. Then $\left\|T_{0}\right\|_{0}=1$. Applying the previous lemma, we construct inductively an increasing sequence $\left(K_{i}\right)_{i=0}^{\infty}$ of finite-dimensional closed subgroups of $E$ and a sequence of linear operators $T_{i}:\left\langle K_{i}\right\rangle \rightarrow L^{2}(0,1), i=0,1,2, \ldots$, such that, for each $i=1,2, \ldots$, the following conditions are satisfied:

$\left(\mathrm{a}_{i}\right) \quad T_{i}\left(K_{i}\right) \subset S_{\mathbb{Z}}(0,1) ;$

(bi) $\quad T_{i \mid\left\langle K_{i-1}\right\rangle}=T_{i-1}$;

(ci $\left.c_{i}\right) \quad\left\|T_{i}\right\|_{0} \leq\left(1+\varepsilon_{i}\right)\left\|T_{i-1}\right\|_{0}$;

$\left(\mathrm{d}_{i}\right)$ there are no linear functionals $f \in\left\langle K_{i}\right\rangle^{*}$ with $f_{\mid\left\langle K_{i-1}\right\rangle} \not \equiv 0$ and $f\left(K_{i}\right) \subset \mathbb{Z}$ such that $\|f\|_{i} \leq 1$.

Define

$$
K_{\infty}=\bigcup_{i=0}^{\infty} K_{i}, \quad K=\bar{K}_{\infty}
$$

(here the closure is taken in the topology of $E$ ).

We proceed to show that the quotient group $\langle K\rangle / K$ is NBT. We first show that $(\langle K\rangle / K)^{\wedge}=\{1\}$. In view of Lemma $5(\mathrm{~b})$, it is enough to prove that there are no non-zero continuous linear functionals $f:\langle K\rangle \rightarrow \mathbb{R}$ with $f(K) \subset \mathbb{Z}$. Suppose that $f$ is such a functional. Then there is $n \in \mathbb{N}$ such that $f_{\mid\left\langle K_{n}\right\rangle} \not \equiv 0$. Since $\left\{B_{i}\right\}_{i=0}^{\infty}$ is a neighbourhood base at zero in $E$, there is $m \in \mathbb{N}$ such that $\|f\|_{m}<1$. Let $i=\max (n+1, m)$ and $f^{\prime}=f_{\mid\left\langle K_{i}\right\rangle}$. Then $f_{\mid\left\langle K_{i-1}\right\rangle}^{\prime} \not \equiv 0, f^{\prime}\left(K_{i}\right) \subset \mathbb{Z}$ and $\left\|f^{\prime}\right\|_{i} \leq 1$, which contradicts $\left(\mathrm{d}_{i}\right)$.

We will now show that the group $\langle K\rangle / K$ admits a non-trivial continuous positive definite function. In view of Lemma $5(\mathrm{a})$, it is enough to show that there is a non-zero continuous linear operator $T:\langle K\rangle \rightarrow L^{2}(0,1)$ with $T(K) \subset L_{\mathbb{Z}}^{2}(0,1)$. Conditions $\left(\mathrm{b}_{i}\right)$ allow us to define a linear operator $T_{\infty}:\left\langle K_{\infty}\right\rangle \rightarrow L^{2}(0,1)$ by $T_{\infty \mid\left\langle K_{i}\right\rangle}=T_{i}$ for every $i=1,2, \ldots$ Conditions $\left(\mathrm{a}_{i}\right)$ imply that $T_{\infty}\left(K_{\infty}\right) \subset S_{\mathbb{Z}}(0,1)$. Conditions $\left(\mathrm{c}_{i}\right)$ imply that $\left\|T_{i}\right\|_{0} \leq \prod_{k=1}^{i}\left(1+\varepsilon_{k}\right)$ for every $i=1,2, \ldots$, so if we denote by $\left\|T_{\infty}\right\|_{0}$ the norm of the operator $T_{\infty}:\left(\left\langle K_{\infty}\right\rangle,\|\cdot\|_{0}\right) \rightarrow L^{2}(0,1)$, we have

$$
\left\|T_{\infty}\right\|_{0}=\sup _{i}\left\|T_{i}\right\|_{0} \leq \prod_{k=1}^{\infty}\left(1+\varepsilon_{k}\right)<\infty .
$$

Let $T:\langle K\rangle \rightarrow L^{2}(0,1)$ be the continuous extension of $T_{\infty}$. Hence

$$
T(K)=T\left(\bar{K}_{\infty}\right) \subset \overline{T\left(K_{\infty}\right)} \subset \overline{S_{\mathbb{Z}}(0,1)}=S_{\mathbb{Z}}(0,1) .
$$


We are now ready to give the proof of Theorem 1 :

Proof of Theorem 1. Let $E$ be a metrizable locally convex space which is not nuclear. According to Lemma 3, there is a continuous seminorm $q$ on $E$ such that if $p \geq q$ is any other continuous seminorm, then

$$
\limsup _{n \rightarrow \infty} n^{1 / 5} \nu_{n}\left(T_{p q}\right)=\infty .
$$

Choose a sequence of seminorms $q=p_{0} \leq p_{1} \leq p_{2} \leq \cdots$ defining the topology of $E$. More precisely, we assume that the sets $B_{k}=\{x \in E$ : $\left.p_{k}(x)<1\right\}, k=0,1, \ldots$, form a neighbourhood base at zero in $E$. Let $F=\left\{x \in E: p_{0}(x)=0\right\}$ and let $\pi: E \rightarrow E / F$ be the canonical projection. Since the sets $\pi\left(B_{k}\right), k=0,1, \ldots$, are convex, symmetric about the origin and do not contain straight lines, their Minkowski functionals are norms. Let us denote them by $\|\cdot\|_{k}$. Let $I_{k}:\left(E / F,\|\cdot\|_{k}\right) \rightarrow\left(E / F,\|\cdot\|_{0}\right)$ be the identity operator. Then for all $n, k=1,2, \ldots$ we have $\nu_{n}\left(I_{k}\right)=\nu_{n}\left(T_{p_{k} p_{0}}\right)$, as is easy to check.

Applying the previous lemma we can find a closed subgroup $L$ of $E / F$ such that the quotient group $\langle L\rangle / L$ is NBT. Define $K=\pi^{-1}(L)$. It is not difficult to see that the quotient group $\langle K\rangle / K$ is NBT.

\section{References}

[1] L. Außenhofer, Contributions to the duality theory of abelian topological groups and to the theory of nuclear groups, Dissertationes Math. 384 (1999).

[2] W. Banaszczyk, Closed subgroups of nuclear spaces are weakly closed, Studia Math. 80 (1984), 119-128.

[3] -, Additive Subgroups of Topological Vector Spaces, Lecture Notes in Math. 1466, Springer, Berlin, 1991.

[4] -, Rearrangement of series in non-nuclear spaces, Studia Math. 107 (1993), $213-222$.

[5] I. M. Gelfand and N. Ya. Vilenkin, Generalized Functions, Vol. 4: Applications of Harmonic Analysis, Academic Press, New York, 1964.

[6] E. Hewitt and K. A. Ross, Abstract Harmonic Analysis, Vol. I, II, Springer, Berlin, 1963, 1970.

[7] J. Lindenstrauss and V. D. Milman, The local theory of normed spaces and its applications to convexity, in: Handbook of Convex Geometry, P. M. Gruber and J. M. Wills (eds.), North-Holland, Amsterdam, 1993, 1149-1220.

[8] D. Mushtari, Certain general questions of the theory of probability measures in linear spaces, Theory Probab. Appl. 18 (1973), 66-77.

[9] - , Probabilities and Topologies in Linear Spaces, Kazan Tracts in Math., Kazan, 1996.

[10] L. A. Santaló, Un invariante afin para los cuerpos convexos del espacio de $n$ dimensiones, Portugal. Math. 8 (1949), 155-161.

[11] M. F. Smith, The Pontrjagin duality theorem in linear spaces, Ann. of Math. 56 (1952), 248-253. 
[12] R. Stegliński, Quotient groups of normed spaces for which the Bochner theorem fails completely, in: Nuclear Groups and Lie Groups (Madrid, 1999), Res. Exp. Math. 24, Heldermann, 2001, 217-225.

[13] V. I. Tarieladze, On the uniqueness theorem for Fourier transform, Trudy Inst. Vychisl. Mat. Akad. Nauk Gruzin. SSR 27 (1987), 195-207 (in Russian).

Faculty of Mathematics

Eódź University

Banacha 22

90-238 Łódź, Poland

E-mail: steglin@math.uni.lodz.pl

Received December 23, 2004

Revised version April 22, 2005 